\title{
Orthogonal decomposition of currents, power definitions and energy transmission in three-phase systems treated in the time domain
}

\author{
Gorazd Štumberger, Boštjan Polajžer, Matej Toman, Drago Dolinar. \\ University of Maribor \\ Faculty of Electrical Engineering and Computer Science \\ Smetanova 17, 2000 Maribor, Slovenia \\ phone:+386 2220 7075, fax:+386 2251 1178, e-mail: Gorazd.Stumberger.@uni-mb.si
}

\begin{abstract}
This paper deals with the orthogonal decomposition of currents in a three-phase system. Currents and voltages of the three-phase system are treated as instantaneous values and as continuous functions defined in given interval. In both cases current and voltage vectors are defined. They are decomposed into two orthogonal components. The first one is collinear with the voltage vector while the second one is orthogonal to it. It is shown, that only the current vector component collinear with the voltage vector contributes to the energy transmission and to active power while the current vector component orthogonal to the voltage vector causes reciprocal energy exchange between source and load whose average value equals zero.
\end{abstract}

Key words: three-phase system, orthogonal current decomposition, power definition, energy transmission, time domain

\section{Introduction}

The authors, who have been working on single-phase two-wire systems over the last years, have soon found and adopted current and voltage definitions, as well as the definitions for instantaneous and active power. The meaning of these definitions is purely physical. They have also adopted the definitions for the reactive and apparent power in systems comprising only the fundamental harmonic components of current and voltage. These definitions of reactive and apparent power are merely constructs without any physical meaning [1], but they nevertheless ensure a satisfactory treatment of some physical phenomena in cases when the current and the voltage comprise the fundamental harmonic components only. However, there is no generally adopted definition of reactive power in single-phase two-wire systems with higher order current and voltage harmonic components. There exist at least two definitions of reactive power, formulated by Budenau and Fryze in 1927 and 1932 to which the authors of [1] to [9] make reference. Budenau's definition, according to which reactive power is the sum of reactive powers of individual harmonic components, has been recognized and accepted. Later, Czarnecki has pointed out the weakness of this definition in [2]. He has shown that in Budenau's definition reactive currents may flow between the sources and the consumers, which causes voltage drops on the internal impedances of sources and unnecessary Joule losses in lines, even in cases when the total reactive power is zero.

Over the last thirty years, current decomposition and the resulting definitions of power have been studied in two ways, i.e. in the time and in the frequency domain. In both cases the authors use the current and the voltage given inside a time window. In the decomposition in the time domain the current and the voltage are expressed as functions of the time, whereas in the decomposition in the frequency domain they are expressed as functions of multiples of the fundamental frequency. Some works dealing with current decomposition, definitions of power, measuring procedures and measures for assessing the quality of energy in single-phase systems are presented in [1] to [6]. Different approaches result in different definitions, which leads to discussions on which definition has more physical ground.

The differences in opinions on various current decomposition procedures and power definitions in single-phase systems are even greater in the case of asymmetrical three-phase systems with higher current and voltage harmonic components. A typical example of such disagreement is the "dispute" between the two well known experts Czarnecki and Emanuel who have different opinions on the physical grounding of some definitions, and who even cannot agree which is more important: power definitions or the orthogonal current decomposition. In spite of the diversity of opinions, powers can be defined and currents can be decomposed in the time and frequency domains of three-phase systems. Some published works on this topic are the papers [7] to [12],[17] and [18].

Power definitions and current decompositions in the frequency and time domains are limited to a selected time window, so they can provide merely an average picture within this window. Therefore, they cannot be used for 
transients, but only for the treatment of steady states. Agaki and Nabae, who worked on the minimization of transmission losses, became aware of this fact in the eighties [13] and [14]. Agaki has introduced the concepts of instantaneous active and reactive power. He has assured the minimization of transmission losses during transients by compensating the instantaneous reactive power without energy storage. In [15], Willems generalized Agaki's definition of the instantaneous reactive power by extending it to any poly-phase system and showed that the decomposition of current in relation to voltage was essential, not the definition of instantaneous reactive power.

In the time of Budenau and Fryze, different power definitions and current decomposition procedures in systems with higher harmonic components were a matter of academic dispute since the currents and the voltages were sinusoidal functions of the fundamental frequency. Today, numerous power electronics devices which can be considered as sources of higher current and voltage harmonic components are connected to the network. This is why current decomposition procedures and power definitions have become a matter of practical interest. They serve as a basis for the minimization of transmission losses, for the development of new measurement techniques and instruments, and also for energy accounts and setting of energy prices [11].

This paper deals with current decomposition, power definitions and energy transmission in the time domain. It makes use of the fact that the currents and voltages of a three-phase system can be handled with vector spaces [16].

\section{Instantaneous values}

Let $u_{l 1}, u_{12}$ and $u_{l 3}$ be the instantaneous values of voltages, and $i_{11}, i_{12}$ and $i_{l 3}$ the instantaneous values of currents in a three-phase system. Let $\mathfrak{B}$ be the orthonormal basis in a three dimensional vector space with an inner product $\langle\bullet, \bullet\rangle$. It is expressed as follows:

$$
\mathfrak{B}=\left\{\mathbf{f}_{l 1}, \mathbf{f}_{l 2}, \mathbf{f}_{l 3}\right\} \quad, \quad\left\langle\mathbf{f}_{j}, \mathbf{f}_{k}\right\rangle=\left\{\begin{array}{lll}
1 & \text { for } & j=k \\
0 & \text { for } & j \neq k
\end{array}\right.
$$

Let us define the current and the voltage vectors by expression (2), which means that one vector from the basis $\mathfrak{B}$ is prescribed to each phase.

$$
\begin{aligned}
& \mathbf{i}:=\mathbf{f}_{l 1} i_{l 1}+\mathbf{f}_{l 2} i_{l 2}+\mathbf{f}_{l 3} i_{l 3} \\
& \mathbf{u}:=\mathbf{f}_{l 1} u_{l 1}+\mathbf{f}_{l 2} u_{l 2}+\mathbf{f}_{l 3} u_{l 3}
\end{aligned}
$$

The current vector is presented in Fig. 1.

Let us define the Euclidean norm by the inner product. The Euclidean norm is a scalar whose value is equal to the vector's length (Fig. 1). The norms of the current and the voltage vector are given by (3).

$$
\begin{aligned}
\|\mathbf{i}\| & :=\sqrt{\langle\mathbf{i}, \mathbf{i}\rangle}=\sqrt{i_{l 1}^{2}+i_{l 2}^{2}+i_{l 3}^{2}} \\
\|\mathbf{u}\| & :=\sqrt{\langle\mathbf{u}, \mathbf{u}\rangle}=\sqrt{u_{11}^{2}+u_{12}^{2}+u_{l 3}^{2}}
\end{aligned}
$$

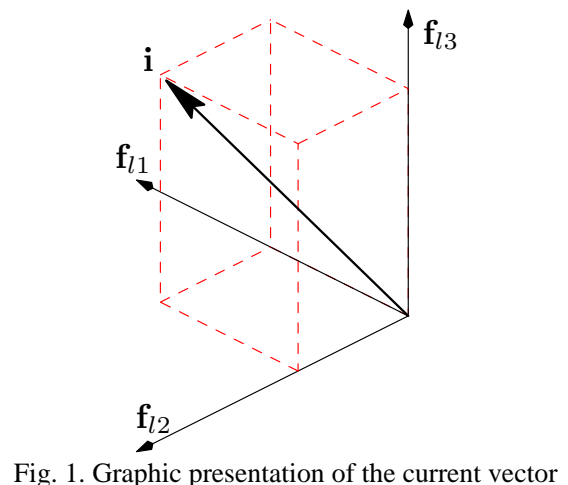

The instantaneous power of a three-phase system is a scalar $p$. Let us define it by the inner product (4). Let its value be equal to the sum of instantaneous power values of all three phases.

$$
p:=\langle\mathbf{u}, \mathbf{i}\rangle=u_{l 1} i_{l 1}+u_{l 2} i_{l 2}+u_{l 3} i_{l 3}
$$

The current vector $\mathbf{i}$ can be expressed as the sum of two vectors, as shown in Fig. 2.

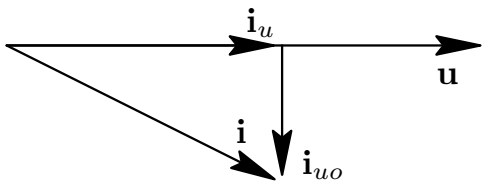

Fig. 2. The current vector, the voltage vector and the orthogonal projections of the current vector

They are the projection of the current vector on the voltage vector described in expression (5), and the orthogonal (perpendicular) projection of the current vector on the voltage vector, as defined in (6):

$$
\begin{gathered}
\mathbf{i}_{u}:=G_{e} \mathbf{u}=\frac{p}{\|\mathbf{u}\|^{2}} \mathbf{u}=\frac{\langle\mathbf{u}, \mathbf{i}\rangle}{\|\mathbf{u}\|^{2}} \mathbf{u} \\
\mathbf{i}_{u о}=\mathbf{i}-\mathbf{i}_{u}
\end{gathered}
$$

Vectors $\mathbf{i}_{u}$ and $\mathbf{i}_{\text {ио }}$ are orthogonal, so their inner product is equal zero.

$$
\left\langle\mathbf{i}_{u}, \mathbf{i}_{\text {ио }}\right\rangle=0
$$

The relations between the current vector norm and the norms of both projections are given by expression (8):

$$
\|\mathbf{i}\|^{2}=\left\|\mathbf{i}_{u}\right\|^{2}+\left\|\mathbf{i}_{\text {uo }}\right\|^{2}
$$

The values of inner products $\left\langle\mathbf{u}, \mathbf{i}_{u}\right\rangle$ and $\langle\mathbf{u}, \mathbf{i}\rangle$ are equal. 


$$
\left\langle\mathbf{u}, \mathbf{i}_{u}\right\rangle=\langle\mathbf{u}, \mathbf{i}\rangle=p
$$

According to (9) the same instantaneous power can be obtained with the vector $\mathbf{i}$ and with the projection $\mathbf{i}_{u}$. The transmission losses in a three-phase system are proportional to $\|\mathbf{i}\|^{2}$. Consequently, minimal losses can be attained when expression (10) is valid.

$$
\mathbf{i}=\mathbf{i}_{u}
$$

In this case the projection iuo $=0$. The current and the voltage vector are collinear, which is the reason why the current in each phase is equal to the voltage multiplied by the constant, regardless of the voltage waveform.

Equation (8) is multiplied by the square of the voltage norm, which gives the equation (11):

$$
\|\mathbf{i}\|^{2}\|\mathbf{u}\|^{2}=\left\|\mathbf{i}_{u}\right\|^{2}\|\mathbf{u}\|^{2}+\left\|\mathbf{i}_{\text {ио }}\right\|^{2}\|\mathbf{u}\|^{2}
$$

Now the instantaneous reactive power $q$ and the instantaneous apparent power $s$ can be defined:

$$
\begin{aligned}
|p| & =\left\|\mathbf{i}_{u}\right\|\|\mathbf{u}\| \\
|q| & :=\left\|\mathbf{i}_{\text {uо }}\right\|\|\mathbf{u}\| \\
|s| & =\|\mathbf{i}\|\|\mathbf{u}\|
\end{aligned}
$$

If we take into account equation (11) and the definitions (12), we get the equation (13) which describes the relations between the defined powers.

$$
s^{2}=p^{2}+q^{2}
$$

\section{Continuous functions in time domain}

This section deals with the orthogonal decompositions of currents and definitions of various powers in the time domain. The currents and the voltages of the three-phase system are treated as continuous functions with a limited frequency spectrum defined in the interval $t \in\left[t_{1}, t_{2}\right]$.

Let $u_{11}(t), u_{12}(t)$ and $u_{13}(t)$ be the voltages and $i_{11}(t)$, $i_{12}(t)$ and $i_{13}(t)$ be the currents of a three-phase system observed inside a selected time window - interval $t \in\left[t_{1}, t_{2}\right]$. The current and the voltage vector are introduced by the definition (14):

$$
\begin{aligned}
\mathbf{i}(t) & :=\mathbf{f}_{l 1} i_{l 1}(t)+\mathbf{f}_{l 2} i_{l 2}(t)+\mathbf{f}_{l 3} i_{l 3}(t) \\
\mathbf{u}(t) & :=\mathbf{f}_{l 1} u_{l 1}(t)+\mathbf{f}_{l 2} u_{l 2}(t)+\mathbf{f}_{l 3} u_{l 3}(t)
\end{aligned}
$$

where $\mathbf{f}_{l 1}, \mathbf{f}_{12}$ and $\mathbf{f}_{l 3}$ are vectors introduced in expression (1).
The inner product is introduced in the vector space of continuous functions by the definition (15):

$$
(\mathbf{u}(t), \mathbf{i}(t)):=\frac{1}{t_{2}-t_{1}} \int_{t_{1}}^{t_{2}}\langle\mathbf{u}(\tau), \mathbf{i}(\tau)\rangle d \tau
$$

which can be expressed also as:

$$
(\mathbf{u}(t), \mathbf{i}(t)):=\frac{1}{t_{2}-t_{1}} \int_{t_{1}}^{t_{2}}\left[u_{l 1}(\tau) \quad u_{l 2}(\tau) \quad u_{l 3}(\tau)\right]\left[\begin{array}{c}
i_{l 1}(\tau) \\
i_{l 2}(\tau) \\
i_{l 3}(\tau)
\end{array}\right] d \tau
$$

Generally, the Euclidean norm, introduced with the inner product, is used in the vector space of functions. In this case definitions (16) are used to introduce the Euclidean norms of the current and the voltage vector:

$$
\begin{aligned}
\|\mathbf{i}(t)\| & :=\sqrt{(\mathbf{i}(t), \mathbf{i}(t))}=\sqrt{I_{l 1}^{2}+I_{l 2}^{2}+I_{l 3}^{2}} \\
\|\mathbf{u}(t)\| & :=\sqrt{(\mathbf{u}(t), \mathbf{u}(t))}=\sqrt{U_{l 1}^{2}+U_{l 2}^{2}+U_{l 3}^{2}}
\end{aligned}
$$

where $I_{l 1}, I_{l 2}, I_{l 3}$ and $U_{l 1}, U_{l 2}, U_{l 3}$ are the RMS values of phase currents and voltages.

In the case of instantaneous values, the current and voltage vectors were introduced by the geometric vectors (Figs. 1 and 2), whereas the lengths of vectors were defined by the norm. In the case of functions the current vector is described by the trajectory made by the current vector in Fig. 1 while the independent variable $t$ runs through the entire interval $\left[t_{1}, t_{2}\right]$.

The norm represents the average distance of the trajectory from the origin of coordinates, measured in the quadratic sense. The square of the current vector norm is equal to the sum of square RMS values of currents in a three-phase system.

The active power, which is a scalar, is introduced by the definition (17). Its value is equal to the sum of active powers of individual phases.

$$
P:=(\mathbf{u}(t), \mathbf{i}(t))=P_{l 1}+P_{l 2}+P_{l 3}
$$

The current vector is expressed as the sum of two vectors in a similar way as it was described for instantaneous values. The first one is called the projection of the current vector on the voltage vector. It is introduced by equation (18). The second one is called the orthogonal projection of the current vector on the voltage vector. It is introduced by definition (19):

$$
\mathbf{i}_{u}(t):=G_{e} \mathbf{u}(t)=\frac{P}{\|\mathbf{u}(t)\|^{2}} \mathbf{u}(t)=\frac{(\mathbf{u}(t), \mathbf{i}(t))}{\|\mathbf{u}(t)\|^{2}} \mathbf{u}(t)
$$

$$
\mathbf{i}_{u o}(t)=\mathbf{i}(t)-\mathbf{i}_{u}(t)
$$


The vectors $\mathbf{i}_{u}(t)$ and $\mathbf{i}_{u о}(t)$ are orthogonal. Their inner product equals zero.

$$
\left(\mathbf{i}_{u}(t), \mathbf{i}_{\text {ио }}(t)\right)=0
$$

The relations between the current vector norm and the norms of both projections are given by equation (21)

$$
\|\mathbf{i}(t)\|^{2}=\left\|\mathbf{i}_{u}(t)\right\|^{2}+\left\|\mathbf{i}_{\text {uo }}(t)\right\|^{2}
$$

The value of the inner product $(\mathbf{u}(t), \mathbf{i}(t))$ is equal to the value of the inner product $\left(\mathbf{u}(t), \mathbf{i}_{u}(t)\right)$

$$
\left(\mathbf{u}(t), \mathbf{i}_{u}(t)\right)=(\mathbf{u}(t), \mathbf{i}(t))=P
$$

If equation (21) is multiplied by the square of the voltage vector norm we get the equation (23). Its terms represent different powers.

$$
\|\mathbf{i}(t)\|^{2}\|\mathbf{u}(t)\|^{2}=\left\|\mathbf{i}_{u}(t)\right\|^{2}\|\mathbf{u}(t)\|^{2}+\left\|\mathbf{i}_{u o}(t)\right\|^{2}\|\mathbf{u}(t)\|^{2}
$$

Expression (22) shows that it is possible to get the same active power in a selected point of the three-phase system with the current vector as well as with the projection $\mathbf{i}_{u}(t)$. According to definition (18) the projection $\mathbf{i}_{u}(t)$ is a vector which is equal to the voltage vector multiplied by the constant $G_{e}=P /\|\mathbf{u}(t)\|^{2}$. Hence it follows that the vectors $\mathbf{i}_{u}(t)$ and $\mathbf{u}(t)$ are collinear. The vector $\mathbf{i}_{u}(t)$ represents the minimal part of the current vector that forms, together with the given voltage vector, the same active power as the whole current vector. The parts of phase currents that belong to the vector $\mathbf{i}_{u}(t)$ are also equal to the phase voltages multiplied by the constant $G_{e}=P /\|\mathbf{u}(t)\|^{2}$ so they have the same phase angle as the belonging phase voltages regardless of their waveform. The projection $\mathbf{i}_{\text {uо }}(t)$ does not affect the active power, but it forms the total current vector $\mathbf{i}(t)$ together with the projection $\mathbf{i}_{u}(t)$. The total Joule losses in lines are proportional to $\|\mathbf{i}(t)\|^{2}$. The unnecessary voltage drops on the internal source impedances are caused by those harmonic components of currents that are not present in voltages. These harmonic components are present only in the projection $\mathbf{i}_{u o}(t)$; they are not present in the projection $\mathbf{i}_{u}(t)$. Therefore it is reasonable to minimize the projection $\mathbf{i}_{u o}(t)$ and thus assure $\mathbf{i}(t)=\mathbf{i}_{u}(t)$. In three-phase systems with unbalanced currents and voltages the transmission losses are greater than necessary even in the presence of condition $\mathbf{i}(t)=\mathbf{i}_{u}(t)$. The RMS values of individual phases are different due to unbalanced operation. Expression (16) shows that the square of the current vector norm equals the sum of square RMS values of phase currents. The norm square will be minimal when the RMS values of all three currents will be equal, which is possible only in case of balanced operation.

On this basis the vector $\mathbf{i}_{u}(t)$ can be called "the active current vector", the vector $\mathbf{i}_{\text {ио }}(t)$ "the reactive power vector" and the vector $\mathbf{i}(t)$ "the apparent current vector". It can be said, by analogy with the aforementioned, that equation (23) describes the relations among the squares of the "apparent", "active" and "reactive" power of a three-phase system.

\section{Results}

In order to demonstrate the impact of current vector $\mathbf{i}(t)$ and its orthogonal components $\mathbf{i}_{u}(t)$ and $\mathbf{i}_{u o}(t)$ on energy transmission, instantaneous powers $p(t), p_{s}(t)$ and $p_{q}(t)$ and their integrals $W(t), W_{s}(t), W_{q}(t)$ are defined by (24) to (29).

$$
\begin{gathered}
p(t):=\left\langle\mathbf{u}(t), \mathbf{i}_{u}(t)\right\rangle \\
p_{s}(t):=\langle\mathbf{u}(t), \mathbf{i}(t)\rangle \\
p_{q}(t):=\left\langle\mathbf{u}(t), \mathbf{i}_{u o}(t)\right\rangle \\
W(t):=W\left(t_{1}\right)+\int_{t_{1}}^{t} p(\tau) d \tau \\
W_{s}(t):=W_{s}\left(t_{1}\right)+\int_{t_{1}}^{t} p_{s}(\tau) d \tau \\
W_{q}(t):=W_{q}\left(t_{1}\right)+\int_{t_{1}}^{t} p_{q}(\tau) d \tau
\end{gathered}
$$

Figure 3 shows three-phase voltages and currents. Current vector components $\mathbf{i}_{u}(t)$ and $\mathbf{i}_{u o}(t)$ in all three phases are shown in Figure 4. Figure 5 shows instantaneous power $p(t), p_{s}(t)$ and $p_{q}(t)$ and energy $W(t), W_{s}(t)$ and $W_{q}(t)$ transmitted due to the current vector $\mathbf{i}(t)$ and its components $\mathbf{i}_{u}(t)$ and $\mathbf{i}_{\text {uо }}(t)$. In the results presented $W\left(t_{1}\right), W_{s}\left(t_{1}\right)$ and $W_{q}\left(t_{1}\right)$ are equal zero.

\section{Conclusion}

The results presented in this work clearly show that only the current vector component $\mathbf{i}_{u}(t)$ contributes to the energy transmission while the component $\mathbf{i}_{\text {ио }}(t)$ causes reciprocal energy exchange between the source and load with average value zero. Thus, the current vector component $\mathbf{i}_{\text {ио }}(t)$ can be compensated in order to minimize transmission losses.

\section{Acknowledgement}

This work was supported in part by the ARRS, Project No. P2-0115. 


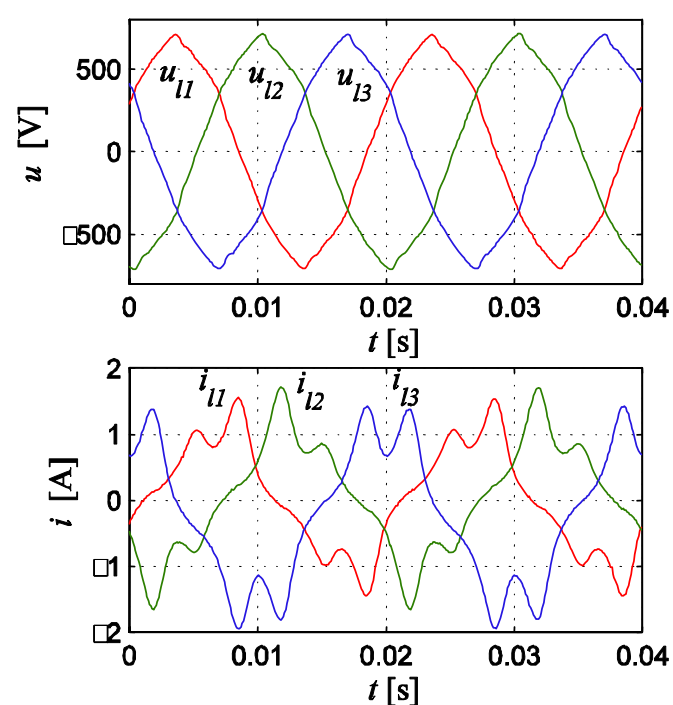

Fig. 3. Three-phase voltages and currents
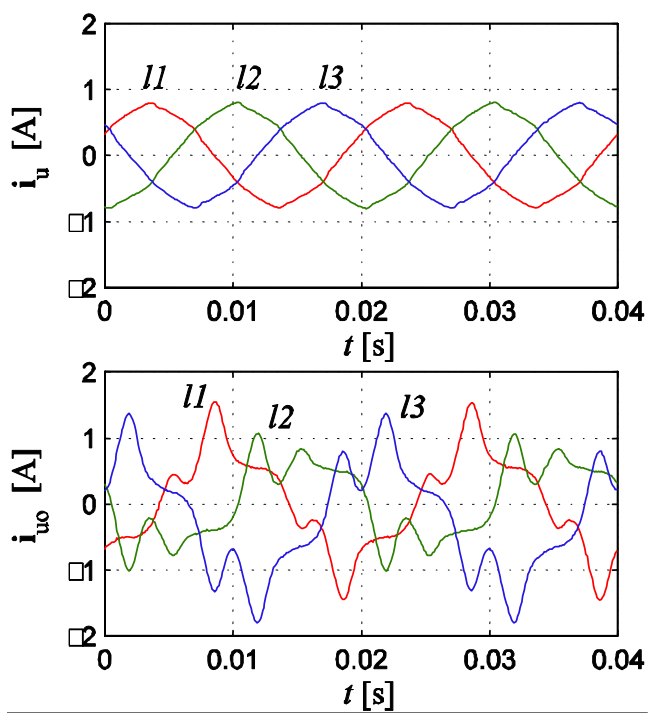

Fig. 4. Components of vectors $\mathbf{i}_{u}(t)$ and $\mathbf{i}_{u o}(t)$ in all three phases
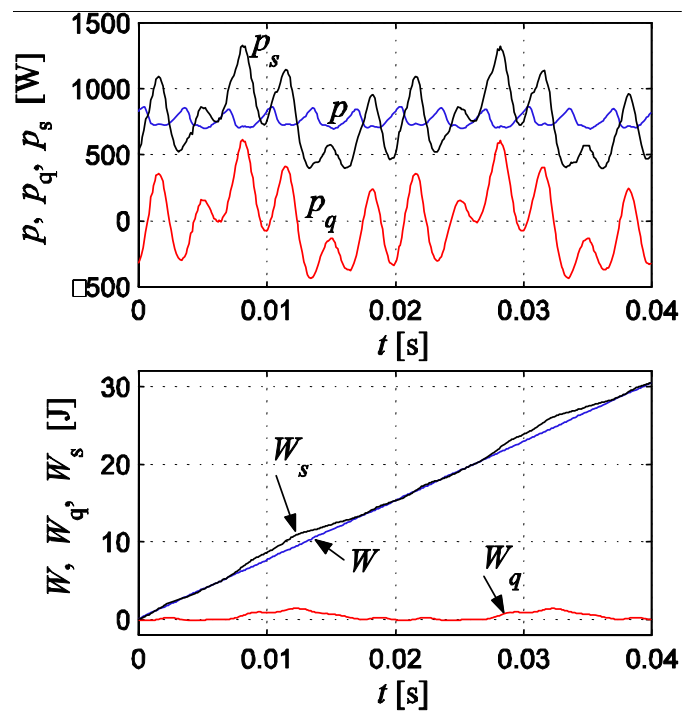

Fig. 5. Instantaneous power and energy due to $\mathbf{i}(t), \mathbf{i}_{u}(t)$ and $\mathbf{i}_{u o}(t)$

\section{References}

[1] S. Svensson, "Power measurement uncertainties in a nonsinusoidal power system," in International Symposium on Electric Power Engineering, Proceedings: Power systems, (Stockholm, Sweden), pp. 617- 622, IEEE / KTH, IEEE, 1995.

[2] L. S. Czarnecki, "What is wrong with the Budeanu concept of reactive and distortion power and why it should be abandoned," IEEE Trans. on Instr. and Meas., vol. 36, no. 3, pp. 834-837, 1987.

[3] L. S. Czarnecki, "Powers in nonsinusoidal networks: their interpretation, analysis, and measurement," IEEE Trans. on Instr. and Meas, vol. 39, no. 2, pp. 340-345, 1990. no. 5, pp. 698-703, 1990.

[5] L. S. Czarnecki, "Scattered and reactive current, voltage, and power in circuits with nonsinusoidal waveforms and their compensation,” IEEE Trans. on Instr. and Meas., vol. 40, no. 3, pp. 563-567, 1991.

[6] R. Sasdelli, G. C. Montanari, "Compensable power for electrical systems in nonsinusoidal conditions," IEEE Trans. on Instr. and Meas., vol. 43, no. 4, pp. 592-598, 1994.

[7] L. S. Czarnecki, "Minimization of unbalanced and reactive currents in three-phase asymmetrical circuits with nonsinusoidal voltage," IEE Proceedings-B, vol. 139, no. 4, pp. 347-354, 1992.

[8] L. S. Czarnecki, "On some misinterpretations of the instantaneous reactive power p-q theory," IEEE Trans. on Power Electronics., vol. 19, no. 3, pp. 828-836, 2004.

[9] A. Ferrero, G. SupertiFurga, "A new approach to the definition of power components in three-phase systems under nonsinusoidal conditions," IEEE Trans. on Instr. and Meas., vol. 40, no. 3, pp. 568-577, 1991.

[10] L. S. Czarnecki, "Power related phenomena in three-phase unbalanced systems," IEEE Trans. on Power Delivery, vol. 10, no. 3, pp. 1168-1176, 1995.

[11] L. S. Czarnecki, "Comments on active power flow and energy accounts in electrical systems with nonsinusoidal waveforms and asymmetry," IEEE Trans. on Power Delivery, vol. 11, no. 3, pp. 1244-1250, 1996.

[12] A. E. Emanuel, "On the definition of power factor and apparent power in unbalanced polyphase circuits with sinusoidal voltage and currents," IEEE Trans. on Power Delivery, vol. 8, no. 3, pp. 841-852, 1993.

[13] H. Akagi, Y. Kanazawa, A. Nabae, "Instantaneous reactive power compensators comprising switching devices without energy storage components," IEEE Trans. on Industry Applications, vol. 20, no. 3, pp. 625-631, 1984.

[14] A. Nabae, T. Tanaka, "A new definition of instantaneous active- reactive current and power based on instantaneous space vectors on polar coordinates in three-phase circuits," IEEE Trans. on Power Delivery, vol. 11, no. 3, pp. 12381243, 1996.

[15] J. L. Willems, “A new interpretation of the Akagi-Nabae power components for nonsinusoidal three-phase situations,” IEEE Trans. on Instr. and Meas., vol. 41, no. 4, pp. 523-527, 1992.

[16] Gorazd Stumberger, Treatment of three-phase systems using vector spaces. Ph.D. Thesis, Faculty of Electrical Engineering and Computer Science, Maribor, 1996.

[17] D. L. Milanez, A. E. Emmanuel, "The instantaneous-space phasor a powerful diagnosis tool," IEEE Trans. on Instr. and Meas., vol. 52, no. 1, pp. 143-148, 2003.

[18] A. E. Emmanuel, "Summary of IEEE Standard 1459: definitions for measurement of electric power quantities under sinusoidal, nonsinusoidal, balanced or unbalanced conditions," IEEE Trans. on Industry Applications, vol. 40, no. 3, pp. 869-876, 2004. 